EPJ Web of Conferences 38, 10002 (2012)

DOI: $10.1051 /$ epjconf/20123810002

(C) Owned by the authors, published by EDP Sciences, 2012

\title{
Self-consistent calculations of quadrupole moments of spherical nuclei
}

\author{
S. Kamerdzhiev ${ }^{1, a}$, S. Krewald ${ }^{2}$, S. Tolokonnikov ${ }^{3,4,}$, E.E. Saperstein ${ }^{3}$, and D.Voitenkov ${ }^{1}$ \\ 1 Institute for Physics and Power Engineering, 249033 Obninsk, Russia \\ 2 Institut für Kernphysik, Forschungszentrum Jülich, D-52425 Jülich, Germany \\ 3 Kurchatov Institute, 123182 Moscow, Russia \\ 4 Moscow Institute of Physics and Technology, 123098 Moscow, Russia
}

\begin{abstract}
The self-consistent Theory of Finite Fermi Systems based on the Energy Density Functional by Fayans et al. with the set DF3-a of parameters fixed previously is used to calculate three kinds of quadrupole moments. At first, we examined systematically quadrupole moments of odd neighbors of semi-magic lead and tin isotopes and $N=50, N=82$ isotones. Second, we found quadrupole moments of the first $2^{+}$states in the same two chains of isotopes. Finally, we evaluated quadrupole moments of odd-odd nuclei neighboring to double magic ones. Reasonable agreement with available experimental data has been obtained. Predictions are made for quadrupole moments of nuclei in the vicinity of unstable magic nuclei
\end{abstract}

\section{Introduction}

As the result of quick development of experimental techniques in nuclear physics, the bulk data on nuclear static moments has become very extensive and comprehensive [1], thus creating a challenge to nuclear theory. First of all, it concerns the nuclei distant from the $\beta$-decay stability valley which are often close to the drip lines and are of great interest to nuclear astrophysics. For this reason, a theoretical approach used for describing such nuclei should have a high predictive power. The self-consistent Theory of Finite Fermi Systems (TFFS) [2] based on the EDF by Fayans et al. [3] is one of such approaches.

A good description of the quadrupole $[4,5]$ and magnetic[6,7] moments of odd semi-magic nuclei has been achieved within this approach. For quadrupole moments, we use a new DF3-a version [8] of the original DF3 functional [3] which was employed in calculations of magnetic moments. It differs from the DF3 version only in the spinorbit parameters $\varkappa, \varkappa^{\prime}$ and the effective tensor force. The DF3-a functional is characterized by a rather strong effective tensor force.

In these calculations, the so-called "singlequasiparticle approximation" has been used, where one quasiparticle in the fixed state $\lambda=(n, l, j, m)$ with the energy $\varepsilon_{\lambda}$ is added to the even-even core. According to the TFFS [9], a quasiparticle differs from a particle of the single-particle model in two respects. First, it possesses the local charge $e_{q}$ and, second, the core is polarized due to the interaction between the particle and the core nucleons via the Landau-Migdal (LM) amplitude. In other words, the quasiparticle possesses the effective charge $e_{\text {eff }}$ caused by the polarizability of the core, which is found by solving the TFFS equations. In the many-particle Shell Model, a similar quantity is introduced as a phenomenological parameter which describes polarizability of the core consisting of outside nucleons. It should be noted that for this series of problems within the scope of the

\footnotetext{
a e-mail: kamerdzhiev@ippe.ru
}

mean-field theory, the self-consistent TFFS is similar to the HF-QRPA approach.

Recently, quadrupole moments of the first $2^{+}$state in even lead and tin isotopes have been found [10], again within the self-consistent TFFS. For this problem which is evidently beyond the mean-field theory, the TFFS results are significantly different from the QRPA ones.

In this paper, we review briefly the results of the cited references on quadrupole moments of odd nuclei and of the $2^{+}$states in even-even ones and add some new calculations for odd unstable nuclei. In addition, we include here some results of [11] for the quadrupole moments of oddodd nuclei neighboring to the double magic ones.

\section{Brief calculation scheme}

The calculation scheme of the self-consistent TFFS based on the EDF method by Fayans et al. is described in detail in Ref. [4]. Here we write down only several formulas which are necessary for understanding main ingredients of the approach. The EDF method by Fayans et al. [3] is a generalization for superfluid finite systems of the original Kohn-Sham EDF method [12]. In this method, the ground state energy of a nucleus is considered as a functional of normal and anomalous densities,

$$
E_{0}=\int \mathcal{E}\left[\rho_{n}(\mathbf{r}), \rho_{p}(\mathbf{r}), v_{n}(\mathbf{r}), v_{p}(\mathbf{r})\right] d^{3} r
$$

Within the TFFS, the static quadrupole moment $Q_{\lambda}$ of an odd nucleus with the odd nucleon in the state $\lambda$ can be found in terms of the diagonal matrix element $\langle\lambda|V(\omega=0)| \lambda\rangle$ of the effective field $V$ in the static external field $V_{0}=\sqrt{16 \pi / 5} r^{2} Y_{20}$. In systems with pairing correlations, equation for the effective field can be written in a compact form as

$$
\hat{V}(\omega)=\hat{e}_{q} V_{0}(\omega)+\hat{\mathcal{F}} \hat{A}(\omega) \hat{V}(\omega),
$$

This is an Open Access article distributed under the terms of the Creative Commons Attribution License 2.0, which permits unrestricted use, distribution, and reproduction in any medium, provided the original work is properly cited. 
where all the terms are matrices. In the standard TFFS notation [9], we have:

$$
\begin{gathered}
\hat{V}=\left(\begin{array}{l}
V \\
d_{1} \\
d_{2}
\end{array}\right), \quad \hat{V}_{0}=\left(\begin{array}{c}
V_{0} \\
0 \\
0
\end{array}\right), \\
\hat{\mathcal{F}}=\left(\begin{array}{ccc}
\mathcal{F} & \mathcal{F}^{\omega \xi} & \mathcal{F}^{\omega \xi} \\
\mathcal{F} \xi \omega & \mathcal{F}^{\xi} & \mathcal{F}^{\xi \omega} \\
\mathcal{F} \xi \omega & \mathcal{F}^{\xi \omega} & \mathcal{F}^{\xi}
\end{array}\right), \\
\hat{A}(\omega)=\left(\begin{array}{ccc}
\mathcal{L}(\omega) & \mathcal{M}_{1}(\omega) & \mathcal{M}_{2}(\omega) \\
\mathcal{O}(\omega) & -\mathcal{N}_{1}(\omega) & \mathcal{N}_{2}(\omega) \\
O(-\omega) & -\mathcal{N}_{1}(-\omega) & \mathcal{N}_{2}(-\omega)
\end{array}\right),
\end{gathered}
$$

where $\mathcal{L}, \mathcal{M}_{1}$, and so on stand for integrals over $\varepsilon$ of the products of different combinations of the Green function $G(\varepsilon)$ and two Gor'kov functions $F^{(1)}(\varepsilon)$ and $F^{(2)}(\varepsilon)$. They can be found in [9].

Isotopic indices in Eqs. (3-5) are omitted for brevity. The explicit form of the above equations is written down for the case of the electric ( $t$-even) symmetry we deal with. In Eq. (4), $\mathcal{F}$ is the usual LM amplitude,

$$
\mathcal{F}=\frac{\delta^{2} \mathcal{E}}{\delta \rho^{2}}
$$

$\mathcal{F}^{\xi}$ is the density-dependent effective pairing interaction,

$$
\mathcal{F}^{\xi}(\rho)=\frac{\delta^{2} \mathcal{E}}{\delta v^{2}}
$$

and the amplitudes $\mathcal{F}^{\omega \xi}=\mathcal{F}^{\xi \omega}$ stand for the mixed second derivatives,

$$
\mathcal{F}^{\omega \xi}=\frac{\delta^{2} \mathcal{E}}{\delta \rho \delta v}
$$

In the case of volume pairing, one has $\mathcal{F}^{\omega \xi}=0$, whereas for the case of surface pairing we deal the amplitude $\mathcal{F}^{\omega \xi}$ is non-zero and should be taken into account when Eqs. (3-5) are solved. As the analysis of Ref. [4] shows, the component $V$ of the vector $\hat{V}$, as a rule, dominates. However, the fields $d_{1}, d_{2}$ also contribute, and sometimes significantly, to the value of $Q_{\lambda}$.

In this article the TFFS equations are solved in the selfconsistent basis obtained within the EDF method with the functional DF3-a. Thus, the same set of parameters has been used to calculate the single particle scheme and, according to Eqs. (6)-(8), the effective interactions in the TFFS equations. We consider the surface kind of pairing as motivated by our previous research [4], see also $a b$ initio arguments in Ref. [13].

\section{Quadrupole moments of odd semi- and near-magic nuclei}

The final expression for the quadrupole moment of an odd nucleus is as follows $[9,14]$ :

$$
Q_{\lambda}^{p, n}=\left(u_{\lambda}^{2}-v_{\lambda}^{2}\right) V_{\lambda}^{p, n},
$$

\begin{tabular}{|c|c|c|c|c|}
\hline nucleus & $\lambda$ & $Q_{\exp }$ & $Q_{\mathrm{th}}$ & $\delta Q$ \\
\hline \multirow[t]{2}{*}{${ }^{39} \mathrm{Ca}$} & $1 d_{3 / 2}$ & $0.036(7)$ & +0.040 & 0.004 \\
\hline & & $0.040(6)$ & & 0.000 \\
\hline \multirow[t]{3}{*}{${ }^{41} \mathrm{Ca}$} & $1 f_{7 / 2}$ & $-0.090(2)$ & -0.078 & 0.012 \\
\hline & & $-0.066(2)$ & & -0.012 \\
\hline & & $-0.080(8)$ & & 0.002 \\
\hline${ }^{85} \mathrm{Kr}$ & $1 g_{9 / 2}$ & $+0.443(3)$ & +0.507 & 0.064 \\
\hline${ }^{87} \mathrm{Kr}$ & $2 d_{5 / 2}$ & $-0.30(3)$ & -0.355 & -0.06 \\
\hline${ }^{87} \mathrm{Sr}$ & $1 g_{9 / 2}$ & $+0.33(2)$ & +0.335 & 0.01 \\
\hline${ }^{89} \mathrm{Sr}$ & $2 d_{5 / 2}$ & $-0.271(9)$ & -0.245 & -0.026 \\
\hline${ }^{89} \mathrm{Zr}$ & $1 g_{9 / 2}$ & $+0.28(10)$ & +0.262 & -0.02 \\
\hline \multirow[t]{3}{*}{${ }^{91} \mathrm{Zr}$} & $2 d_{5 / 2}$ & $-0.176(3)$ & -0.195 & -0.019 \\
\hline & & $(-) 0.257(13)$ & & 0.062 \\
\hline & & $-0.206(10)$ & & 0.011 \\
\hline${ }^{109} \mathrm{Sn}$ & $2 d_{5 / 2}$ & $+0.31(10)$ & +0.250 & -0.06 \\
\hline${ }^{111} \mathrm{Sn}$ & $1 g_{7 / 2}$ & $+0.18(9)$ & +0.029 & -0.13 \\
\hline${ }^{115} \mathrm{Sn}$ & $1 g_{7 / 2}^{*}$ & $0.26(3)$ & +0.377 & 0.12 \\
\hline \multirow[t]{3}{*}{${ }^{119} \mathrm{Sn}$} & $2 d_{3 / 2}^{*}$ & $0.094(11)$ & -0.035 & -0.129 \\
\hline & & $-0.065(5)$ & & 0.030 \\
\hline & & $-0.061(3)$ & & 0.026 \\
\hline${ }^{121} \mathrm{Sn}$ & $2 d_{3 / 2}$ & $-0.02(2)$ & +0.063 & 0.08 \\
\hline${ }^{135} \mathrm{Xe}$ & $2 d_{3 / 2}$ & $+0.214(7)$ & +0.217 & 0.003 \\
\hline${ }^{137} \mathrm{Xe}$ & $2 f_{7 / 2}$ & $-0.48(2)$ & -0.376 & 0.10 \\
\hline${ }^{137} \mathrm{Ba}$ & $2 d_{3 / 2}$ & $+0.245(4)$ & +0.254 & 0.009 \\
\hline${ }^{139} \mathrm{Ba}$ & $2 f_{7 / 2}$ & $-0.573(13)$ & -0.445 & 0.128 \\
\hline${ }^{141} \mathrm{Nd}$ & $2 d_{3 / 2}$ & $+0.32(13)$ & +0.289 & -0.03 \\
\hline${ }^{143} \mathrm{Nd}$ & $2 f_{7 / 2}$ & $-0.61(2)$ & -0.518 & 0.09 \\
\hline${ }^{143} \mathrm{Sm}$ & $2 d_{3 / 2}$ & $+0.4(2)$ & +0.296 & 0.1 \\
\hline${ }^{145} \mathrm{Sm}$ & $2 f_{7 / 2}$ & $-0.60(7)$ & -0.537 & 0.06 \\
\hline${ }^{197} \mathrm{~Pb}$ & $3 p_{3 / 2}$ & $-0.08(17)$ & +0.195 & 0.27 \\
\hline${ }^{199} \mathrm{~Pb}$ & $3 p_{3 / 2}$ & $+0.08(9)$ & +0.272 & 0.19 \\
\hline${ }^{201} \mathrm{~Pb}$ & $2 f_{5 / 2}$ & $-0.01(4)$ & +0.137 & 0.15 \\
\hline${ }^{203} \mathrm{~Pb}$ & $2 f_{5 / 2}$ & $+0.10(5)$ & +0.284 & 0.18 \\
\hline${ }^{205} \mathrm{~Pb}$ & $2 f_{5 / 2}$ & $+0.23(4)$ & +0.336 & 0.09 \\
\hline${ }^{209} \mathrm{~Pb}$ & $2 g_{9 / 2}$ & $-0.3(2)$ & -0.264 & 0.1 \\
\hline${ }^{211} \mathrm{~Pb}$ & $2 g_{9 / 2}$ & $+0.09(6)$ & -0.283 & -0.37 \\
\hline \multirow[t]{2}{*}{${ }^{113} \mathrm{Sn}$} & $1 h_{11 / 2}^{*}$ & 0.41(4) & -0.776 & -0.37 \\
\hline & & $0.48(5)$ & & -0.30 \\
\hline${ }^{115} \mathrm{Sn}$ & $1 h_{11 / 2}^{*}$ & $0.38(6)$ & -0.703 & -0.32 \\
\hline${ }^{117} \mathrm{Sn}$ & $1 h_{11 / 2}^{*}$ & $-0.42(5)$ & -0.593 & -0.17 \\
\hline${ }^{119} \mathrm{Sn}$ & $1 h_{11 / 2}^{*}$ & $0.21(2)$ & -0.469 & -0.25 \\
\hline${ }^{121} \mathrm{Sn}$ & $1 h_{11 / 2}^{*}$ & $-0.14(3)$ & -0.293 & -0.15 \\
\hline${ }^{123} \mathrm{Sn}$ & $1 h_{11 / 2}^{11 / 2}$ & $+0.03(4)$ & -0.123 & -0.15 \\
\hline${ }^{125} \mathrm{Sn}$ & $1 h_{11 / 2}$ & $+0.1(2)$ & +0.039 & -0.1 \\
\hline${ }^{135} \mathrm{Xe}$ & $1 h_{11 / 2}^{*}$ & $+0.62(2)$ & +0.504 & 0.12 \\
\hline${ }^{137} \mathrm{Ba}$ & $1 h_{11 / 2}^{*}$ & $+0.78(9)$ & +0.588 & -0.19 \\
\hline${ }^{147} \mathrm{Gd}$ & $1 i_{13 / 2}^{*}$ & $-0.73(7)$ & -0.791 & -0.06 \\
\hline${ }^{191} \mathrm{~Pb}$ & $1 i_{13 / 2}^{*}$ & $+0.085(5)$ & +0.0004 & -0.085 \\
\hline${ }^{193} \mathrm{~Pb}$ & $1 i_{13 / 2}^{*}$ & $+0.195(10)$ & +0.335 & 0.140 \\
\hline${ }^{195} \mathrm{~Pb}$ & $1 i_{13 / 2}^{*}$ & $+0.306(15)$ & +0.689 & 0.383 \\
\hline${ }^{197} \mathrm{~Pb}$ & $1 i_{13 / 2}^{*}$ & $+0.38(2)$ & +0.980 & 0.60 \\
\hline${ }^{205} \mathrm{~Pb}$ & $1 i_{13 / 2}^{*}$ & $0.30(5)$ & +0.665 & 0.37 \\
\hline
\end{tabular}

where $u_{\lambda}, v_{\lambda}$ are the Bogolyubov coefficients and

$$
V_{\lambda}=-\frac{2 j-1}{2 j+2} \int V(r) R_{n l j}^{2}(r) r^{2} d r .
$$

Table 1. Quadrupole moments $Q(e b)$ of odd-neutron nuclei in the state $\lambda$.

For odd neighbors of a magic nucleus the "Bogolyubov" factor in (9) reduces to 1 for a particle state and to -1 for a hole one, see also [15]. If the odd nucleon belongs to the superfluid component, the factor $\left(u_{\lambda}^{2}-v_{\lambda}^{2}\right)$ in Eq. (9) becomes non-trivial. It changes permanently depending on the state $\lambda$ and the nucleus under consideration. This factor determines the sign of the quadrupole moment. It depends essentially on values of the single-particle basis energies 
$\varepsilon_{\lambda}$ reckoned from the chemical potential $\mu$ as we have

$$
\left(u_{\lambda}^{2}-v_{\lambda}^{2}\right)=\left(\varepsilon_{\lambda}-\mu\right) / E_{\lambda},
$$

$E_{\lambda}=\sqrt{\varepsilon_{\lambda}^{2}+\Delta_{\lambda}^{2}}$. Keeping in mind such sensitivity, we found this quantity for a given odd nucleus $(Z, N+1)$ or $(Z+1, N), N, Z$ even, with taking into account the blocking effect in the pairing problem [14] putting the odd nucleon to the state $\lambda$ under consideration. For the $V_{\lambda}$ value in Eq. (9) we used the half-sum of these values in two neighboring even nuclei.

The results of the calculations are presented in Tables 1 and 2 which contain odd-neutron and odd-proton nuclei respectively with known experimental quadrupole moments (our predictions for odd nuclei with unknown quadrupole moments see in $[4,5])$. One can see that the theoretical sign of the quadrupole moment is correct in all cases when the sign of the experimental moment is known. This permits to use our predictions to determine the sign when it is unknown. Several rather strong disagreements with the experimental data for high-j levels $1 h_{11 / 2}$ in $\mathrm{Sn}$ isotopes and $1 i_{13 / 2}$ in $\mathrm{Pb}$ isotopes originate from their too distant positions from the Fermi level, see [5], where it was found that the $Q$ values depend strongly on the single-particle level structure. It follows mainly from Eq. (11).

To evaluate the agreement with experiment quantitatively, we calculate the mean theory-experiment difference

$$
\sqrt{\overline{(\delta Q)_{\mathrm{rms}}^{2}}}=\sqrt{\frac{1}{\mathcal{N}} \sum_{i}\left(Q_{i}^{\mathrm{th}}-Q_{i}^{\exp }\right)^{2}},
$$

with obvious notation. On average, the agreement, can be considered as reasonable. For 42 quadrupole moments of odd-neutron nuclei, the average disagreement between theory and experiment is not so small, $\sqrt{\overline{(\delta Q)_{\mathrm{rms}}^{2}}}=0.189 \mathrm{e} \mathrm{b}$. However, it is concentrated mainly in 15 intruder states for which we have $\sqrt{(\delta Q)_{\mathrm{rms}}^{2}}$ [intruder $]=0.269 \mathrm{e} \mathrm{b}$. For the rest of 27 "normal" states, the disagreement is rather moderate $\sqrt{\overline{(\delta Q)_{\mathrm{rms}}^{2}}}$ [normal] $=0.125$ e b. For protons, agreement is worse. The rms deviation is $\sqrt{\overline{(\delta Q)_{\mathrm{rms}}^{2}}}=0.254 \mathrm{e} \mathrm{b}$. The main contribution to this deviation comes from In and $\mathrm{Sb}$ isotopes, odd neighbors of even tin nuclei. It is the result of too strong quadrupole field $V_{n, p}(r)$ for the DF3-a functional [4]. For neutrons, this drawback is partially hidden with multiplying by the Bogolyubov factor, but for protons it appears to the full extent. For more detailed discussion, see [5].

For odd-neutron neighbors of even $N=50$ isotones, the proton-subsystem is superfluid and the neutron Bogolyubov factor in Eq. (9) is \pm 1 . In this case, agreement with the data is almost perfect, $\sqrt{\overline{(\delta Q)_{\mathrm{rms}}^{2}}}=0.041 \mathrm{e} \mathrm{b.} \mathrm{The}$ situation is similar for odd-neutron neighbors of even isotones with $N=82$. Again, agreement with the data is rather good, $\sqrt{\overline{(\delta Q)_{\mathrm{rms}}^{2}}}=0.093 \mathrm{e} \mathrm{b}$.

For the major part of nuclei in Tables 1 and 2, neighboring to double-magic ones, let us call them "near-magic", the quality of agreement is rather good. Therefore, we hope to predict reasonably the quadrupole moment values for such nuclei including strongly proton- or neutron-rich ones. These predictions are presented in Table 3.
Table 2. Quadrupole moments $Q$ (b) of odd-proton nuclei in the

\begin{tabular}{|c|c|c|c|c|}
\hline nucl. & $\lambda$ & $Q_{\exp }$ & $Q_{\text {th }}$ & $\delta Q$ \\
\hline${ }^{39} \mathrm{~K}$ & $1 d_{3 / 2}$ & $0.0585(6)$ & 0.069 & 0.010 \\
\hline \multirow[t]{3}{*}{${ }^{41} \mathrm{Sc}$} & $1 f_{7 / 2}$ & $-0.156(3)$ & -0.139 & 0.017 \\
\hline & & $0.120(6)$ & & -0.019 \\
\hline & & $0.168(8)$ & & 0.029 \\
\hline \multirow[t]{2}{*}{${ }^{87} \mathrm{Rb}$} & $2 p_{3 / 2}$ & $+0.134(1)$ & +0.132 & -0.002 \\
\hline & & $+0.138(1)$ & & -0.006 \\
\hline${ }^{105} \mathrm{In}$ & $1 g_{9 / 2}$ & $+0.83(5)$ & +0.833 & 0.00 \\
\hline${ }^{107} \mathrm{In}$ & $1 g_{9 / 2}$ & $+0.81(5)$ & +0.976 & 0.17 \\
\hline${ }^{109} \mathrm{In}$ & $1 g_{9 / 2}$ & $+0.84(3)$ & +1.113 & 0.27 \\
\hline${ }^{111} \mathrm{In}$ & $1 g_{9 / 2}$ & $+0.80(2)$ & +1.165 & 0.36 \\
\hline${ }^{113} \mathrm{In}$ & $1 g_{9 / 2}$ & $+0.80(4)$ & +1.117 & 0.32 \\
\hline \multirow{2}{*}{${ }^{115} \mathrm{In}$} & $1 g_{9 / 2}$ & $+0.81(5)$ & +1.034 & 0.22 \\
\hline & & $0.58(9)$ & & 0.45 \\
\hline${ }^{117} \mathrm{In}$ & $1 g_{9 / 2}$ & $+0.829(10)$ & +0.965 & 0.136 \\
\hline${ }^{119} \mathrm{In}$ & $1 g_{9 / 2}$ & $+0.854(7)$ & +0.909 & 0.055 \\
\hline${ }^{121} \mathrm{In}$ & $1 g_{9 / 2}$ & $+0.814(11)$ & +0.833 & 0.019 \\
\hline${ }^{123} \mathrm{In}$ & $1 g_{9 / 2}$ & $+0.757(9)$ & +0.743 & -0.014 \\
\hline${ }^{125} \mathrm{In}$ & $1 g_{9 / 2}$ & $+0.71(4)$ & +0.663 & -0.05 \\
\hline${ }^{127} \mathrm{In}$ & $1 g_{9 / 2}$ & $+0.59(3)$ & +0.550 & -0.04 \\
\hline${ }^{115} \mathrm{Sb}$ & $2 d_{5 / 2}$ & $-0.36(6)$ & -0.882 & -0.52 \\
\hline${ }^{119} \mathrm{Sb}$ & $2 d_{5 / 2}$ & $-0.37(6)$ & -0.766 & -0.40 \\
\hline \multirow[t]{2}{*}{${ }^{121} \mathrm{Sb}$} & $2 d_{5 / 2}$ & $-0.36(4)$ & -0.721 & -0.36 \\
\hline & & $-0.45(3)$ & & -0.27 \\
\hline${ }^{123} \mathrm{Sb}$ & $1 g_{7 / 2}$ & $-0.49(5)$ & -0.739 & -0.25 \\
\hline${ }^{137} \mathrm{Cs}$ & $1 g_{7 / 2}$ & $+0.051(1)$ & -0.031 & -0.080 \\
\hline${ }^{139} \mathrm{La}$ & $1 g_{7 / 2}$ & $+0.20(1)$ & +0.103 & -0.10 \\
\hline \multirow[t]{2}{*}{${ }^{141} \mathrm{Pr}$} & $2 d_{5 / 2}$ & $-0.077(6)$ & -0.120 & -0.043 \\
\hline & & $-0.059(4)$ & & -0.061 \\
\hline${ }^{145} \mathrm{Eu}$ & $2 d_{5 / 2}$ & $+0.29(2)$ & +0.156 & -0.13 \\
\hline${ }^{205} \mathrm{Tl}$ & $3 d_{3 / 2}^{*}$ & $+0.74(15)$ & +0.227 & -0.51 \\
\hline \multirow[t]{2}{*}{${ }^{203} \mathrm{Bi}$} & $1 h_{9 / 2}$ & $-0.93(7)$ & -1.323 & -0.39 \\
\hline & & $-0.68(6)$ & & -0.64 \\
\hline \multirow[t]{2}{*}{${ }^{205} \mathrm{Bi}$} & $1 h_{9 / 2}$ & $-0.81(3)$ & -0.945 & -0.14 \\
\hline & & $-0.59(4)$ & & -0.36 \\
\hline \multirow[t]{3}{*}{${ }^{207} \mathrm{Bi}$} & $1 h_{9 / 2}$ & $-0.76(2)$ & -0.454 & 0.31 \\
\hline & & $-0.55(4)$ & & 0.10 \\
\hline & & $-0.60(11)$ & & 0.15 \\
\hline \multirow[t]{6}{*}{${ }^{209} \mathrm{Bi}$} & $1 h_{9 / 2}$ & $-0.516(15)$ & -0.342 & 0.18 \\
\hline & & $-0.37(3)$ & & 0.03 \\
\hline & & $-0.55(1)$ & & 0.21 \\
\hline & & $-0.77(1)$ & & 0.43 \\
\hline & & $-0.40(5)$ & & 0.06 \\
\hline & & $-0.39(3)$ & & 0.05 \\
\hline \multirow[t]{2}{*}{${ }^{213} \mathrm{Bi}$} & $1 h_{9 / 2}$ & $-0.83(5)$ & -0.508 & 0.32 \\
\hline & & $-0.60(5)$ & & 0.09 \\
\hline
\end{tabular}
state $\lambda$.

As it was mentioned in Introduction, the core polarizability by the quadrupole external field is characterized directly by the effective quadrupole charges, which are defined naturally within TFFS as $e_{\mathrm{eff}}^{p, n}=V_{\lambda}^{p, n} /\left(V_{0}^{p}\right)_{\lambda}$ [16]. In Tables 1 and 2 , there are only two nucleus, ${ }^{209} \mathrm{Bi}$ and ${ }^{209} \mathrm{~Pb}$, with a double-magic core. In this case, the polarizability is relatively moderate, $e_{\mathrm{eff}}^{p}=1.4, e_{e f f}^{n}=0.9$. In nuclei with unfilled neutron shell, it becomes much stronger, $e_{\text {eff }} \simeq 3 \div 6$ [4]. The reason is rather obvious. Indeed, for the case of positive parity field $V_{0}$, virtual transitions inside the unfilled shell begin to contribute in such nuclei and small energy denominators appear in the propagator $\mathcal{L}^{n}$, Eq. (5), playing the main role in Eq. (2) for the problem under consideration. This enhances the neutron response to the field $V_{0}$ and, via the strong LM neutron-proton interaction am- 
Table 3. Predictions for quadrupole moments $Q \quad(e b)$ of odd near-magic nuclei.

\begin{tabular}{|c|c|c|c|c|}
\hline nucl. & $J^{\pi}$ & $T_{1 / 2}$ & $Q_{\text {theor }}$ & $Q_{\exp }$ \\
\hline${ }_{25}^{55} \mathrm{Ni}_{27}$ & $7 / 2^{+}$ & $204.7 \mathrm{~ms}$ & -0.26 & - \\
\hline $\begin{array}{l}{ }_{28}^{28} \mathrm{Ni}_{29} \\
\end{array}$ & $3 / 2^{-}$ & $35.6 \mathrm{~h}$ & -0.17 & - \\
\hline${ }_{28}^{77} \mathrm{Ni}_{49}$ & $(9 / 2)^{+}$ & $128 \mathrm{~ms}$ & 0.20 & - \\
\hline${ }_{29}^{28} \mathrm{Ni}_{50}$ & $\left(5 / 2^{+}\right)$ & $635 \mathrm{~ns}$ & -0.12 & - \\
\hline${ }_{50}^{101} \mathrm{Sn}_{51}$ & $(5 / 2)^{+}$ & $1.7 \mathrm{~s}$ & -0.21 & - \\
\hline${ }_{50}^{131} \mathrm{Sn}_{81}$ & $\left(3 / 2^{+}\right)$ & $56 \mathrm{~s}$ & 0.10 & $-0.04(8)$ \\
\hline${ }_{50}^{133} \mathrm{Sn}_{83}$ & $7 / 2^{-}$ & $1.46 \mathrm{~s}$ & -0.17 & - \\
\hline${ }_{82}^{207} \mathrm{~Pb}_{125}$ & $(1 / 2)^{-}$ & stable & 0 & - \\
\hline${ }_{27}^{82} \mathrm{Co}_{28}$ & $7 / 2^{-}$ & $17.53 \mathrm{~h}$ & 0.31 & - \\
\hline${ }_{29}^{5} \mathrm{Cu}_{28}$ & $3 / 2^{-}$ & $196.3 \mathrm{~ms}$ & -0.20 & - \\
\hline${ }_{29}^{79} \mathrm{Cu}_{50}$ & $\left(3 / 2^{-}\right)$ & $188 \mathrm{~ms}$ & -0.13 & - \\
\hline${ }_{49}^{99} \mathrm{In}_{50}$ & $(9 / 2)^{+}$ & $3 \mathrm{~s}$ & -0.35 & - \\
\hline${ }_{49}^{4} \mathrm{In}_{82}$ & $(9 / 2)^{+}$ & $0.28 \mathrm{~s}$ & 0.28 & - \\
\hline${ }_{51}^{49} \mathrm{Sb}_{82}$ & $(7 / 2)^{+}$ & $2.34 \mathrm{~m}$ & -0.23 & - \\
\hline${ }_{81}^{207} \mathrm{Tl}_{126}$ & $1 / 2^{+}$ & $4.77 \mathrm{~m}$ & 0 & - \\
\hline
\end{tabular}

plitude $\mathcal{F}^{n p}$, the proton response as well. The results in Table 2 for the chain ${ }^{203,205,209} \mathrm{Bi}$ show how the polarizability grows with increase of the number of neutron holes. Keeping in mind this physics, one can represent the effective charges as $e_{\mathrm{eff}}^{p}=1+e_{\mathrm{pol}}^{p}, e_{\mathrm{eff}}^{n}=e_{\mathrm{pol}}^{n}$ where $e_{\mathrm{pol}}^{p, n}$ is the pure polarizability charge. To separate contributions of the unfilled shells and core nucleons explicitly, one can divide the Hilbert space of the QRPA equations (2) to the "valent" and subsidiary ones and carry out the corresponding renormalization procedure [17].

\section{Quadrupole moments of the first $2^{+}$ states in $\mathrm{Sn}$ and $\mathrm{Pb}$ isotopes}

Account for the phonon coupling (PC) is the direct way to generalize the standard nuclear theory. As a rule, the so-called $g^{2}$ approximation is used where $g$ is the phonon creation amplitude. However, almost all of these generalizations did not take into account all the $g^{2}$ terms, limiting themselves with the mass operator pole diagrams only, see the first diagram in figure 1, where diagrams for the mass operator are displayed. The second diagram represents the sum of all $g^{2}$ non-pole diagrams usually called the tadpole. The problem of consistent consideration of all $g^{2}$ terms including tadpoles was analyzed firstly in the article by Khodel [18]. The method developed was applied to magic nuclei, mainly for ground state nuclear characteristics, within the self-consistent TFFS [2]. It was found that, as a rule, the tadpole contributions in magic nuclei are noticeable and are often of opposite sign as compared with those of the pole terms. The first attempts to include phonon tadpole effects for nuclei with pairing were recently made in Refs. [10,19,20].

According to Ref. [18], in the $g^{2}$ approximation, the matrix element $M_{L L}$ for a static moment of the excited state (phonon) with the orbital angular moments $L$ in a static external field $V^{0}$ is determined in terms of the change of the one-particle Green function (GF) in the field of this

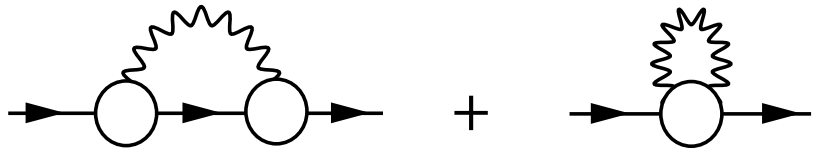

Fig. 1. $g^{2}$ order corrections to the mass operator in magic nuclei. The circles with one wavy line in the first term are the phonon creation amplitudes $g$. The second term is the phonon tadpole.

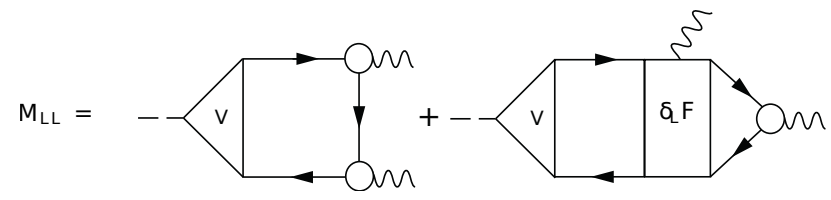

Fig. 2. Matrix element $M_{L L}$ in the form of Eq. (16).

phonon:

$$
\begin{gathered}
M_{L L}=\int V^{0}(\mathbf{r}) \delta_{L L}^{(2)} G(\mathbf{r}, \mathbf{r}, \varepsilon) d \mathbf{r} \frac{d \varepsilon}{2 \pi l}, \\
\delta_{L L}^{(2)} G=\delta_{L}\left(G g_{L} G\right)=G(\varepsilon) g_{L} G\left(\varepsilon+\omega_{L}\right) g_{L} G(\varepsilon) \\
+G(\varepsilon) g_{L} G\left(\varepsilon-\omega_{L}\right) g_{L} G(\varepsilon)+G(\varepsilon) \delta_{L} g_{L} G(\varepsilon),
\end{gathered}
$$

where $g_{L}$ is the amplitude for the production of the $\mathrm{L}$ phonon with the energy $\omega_{L}$ and $\delta_{L} g_{L}$ is the variation of $g_{L}$ in the field of other $L$ phonon. This quantity is the main part of the phonon tadpole in figure 1. After some transformations of these expressions one can obtain

$$
M_{L L}=V^{0} G g_{L} G g_{L} G+V^{0} A \delta_{L} g_{L}
$$

It is convenient to transform this expression in such a way that the effective field $V$, Eq. (2), appears instead of the external field $V^{0}$. After regrouping terms in Eq. (15) and in the integral equation for $\delta_{L} g_{L}$, for details, see Refs. $[2,10,20]$, we obtain the ultimate expression,

$$
M_{L L}=V G g_{L} G g_{L} G+V A \delta_{L} \mathcal{F} A g_{L},
$$

which is illustrated in figure 2 . It contains now the effective field $V$, Eq. (2), instead of $V^{0}$ and the quantity $\delta_{L} \mathcal{F}$ in the second term which denotes the variation of the effective ph interaction $\mathcal{F}$ in the field of the $L$ phonon. For the density dependent TFFS effective interaction $\mathcal{F}(\rho)$, the following ansatz can be readily obtained $[2,18]$ :

$$
\delta_{L} \mathcal{F}(\mathbf{r})=\frac{\partial \mathcal{F}}{\partial \rho} \rho_{L}^{\operatorname{tr}}(r) Y_{L M}(\mathbf{n}),
$$

where $\rho_{L}^{\mathrm{tr}}=A g_{L}$ is the transition density for the $L$ phonon excitation. The first term of Eq. (16) coincides with the result of Refs. [21,22] while the second one, with the $\delta_{L} \mathcal{F}$ quantity, is a generalization to take into account all the $g^{2}$ terms.

All the above equations can be readily modified for such processes as the transition between the excited states $L$ and $L^{\prime}$ in the external field $V^{0}\left(\omega=\omega_{L^{\prime}}-\omega_{L}\right)$ or the excitation of the two-phonon state $L+L^{\prime}$ in the external field $V^{0}\left(\omega=\omega_{L^{\prime}}+\omega_{L}\right)$. The static moment case corresponds to $\omega=0, \omega_{L^{\prime}}=\omega_{L}$.

This approach for magic nuclei has been generalized for non-magic ones in $[10,23]$. Then eight matrix elements 


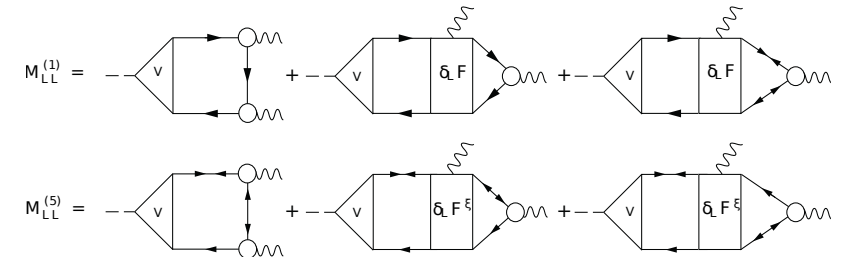

Fig. 3. Matrix elements for $M_{L L}^{(1)}$ and $M_{L L}^{(5)}$ for non-magic nuclei.

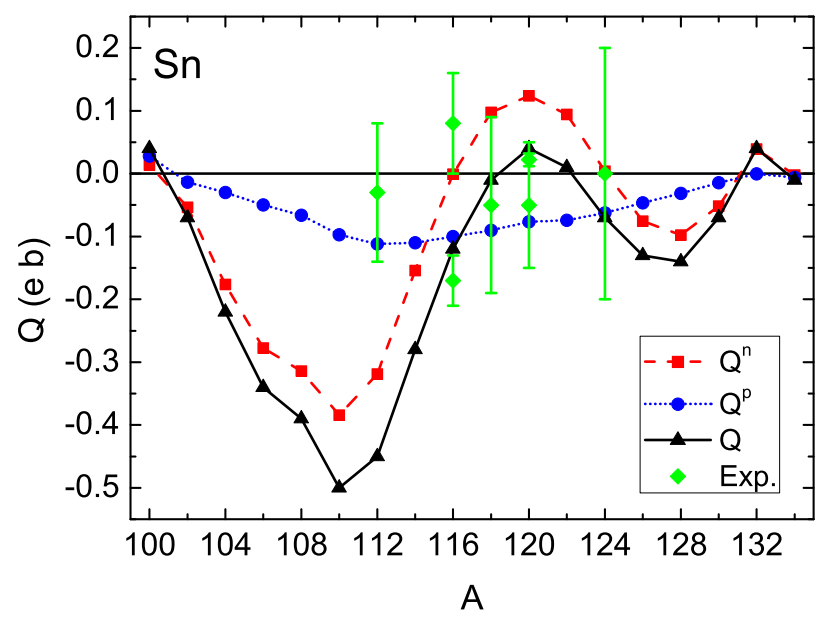

Fig. 4. Quadrupole moments of the first $2+$ excited states in even Sn isotopes.

instead of one in figure 2 should be considered, two of them are shown in figure 3.

It is necessary to compare this expression with the QRPA approach. Here we mean the usual scheme [24] which uses the QRPA wave functions for the matrix element between two excited states. In Ref. [24] the expression for the $B(E 2)$ quantity has been derived using the bare external field $V^{0}$ and the QRPA wave functions without the pp and hh-channels. The analytical expression for the sum of the eight above-mentioned matrix elements consists of two parts. The first part coincides with the corresponding formula in $[14,24]$ with one important correction, which is the first generalization of the QRPA approach. Namely, instead of the external field $V^{0}$, which does not depend on the frequency, the effective field $V$ appears, which depends in general on the frequency $\omega=\omega_{L} \pm \omega_{L}^{\prime}$. The second part of the sum is new and describes the contribution of the ground state correlations (GSC), the so-called backwardgoing diagrams, to the first diagrams of figure 3 with the integrals of three GF's ("triangle"). This is the second generalization. We calculate the contribution of such correlations separately. The terms with $\delta_{L} \mathcal{F}$ and $\delta_{L} \mathcal{F}^{\xi}$, figure 3, are the third generalization of the QRPA approach. Note that these terms are also absent in Refs. [21,22,25-27]. The main difference of our approach from the calculations in Refs. [22,25-27] is the self-consistency on the (Q)RPA level and absence of any phenomenological or fitted parameters.

We calculated the quadrupole moments of the first $2^{+}$ states in the tin and lead isotopes in the $\lambda$-representation with self-consistent single-particle wave functions $\phi_{\lambda}$ obtained within the EDF method of Ref. [3] with the functional DF3-a [8]. A spherical box of the radius $R=16 \mathrm{fm}$ is used to simulate the single-particle continuum. We examined the dependence of the results on the cut-off energy
Table 4. Quadrupole moments $Q(e b)$ of the first $2^{+}$states in $\mathrm{Sn}$ and $\mathrm{Pb}$ isotopes.

\begin{tabular}{|c|c|c|c|c|}
\hline nucl. & $Q_{\text {theor }}$ & $Q_{\exp }[1]$ & $Q(G S C=0)$ & $Q_{\mathrm{QRPA}}$ \\
\hline${ }^{100} \mathrm{Sn}$ & 0.04 & - & 0.05 & 0.017 \\
\hline${ }^{102} \mathrm{Sn}$ & -0.07 & - & -0.02 & -0.001 \\
\hline${ }^{104} \mathrm{Sn}$ & -0.22 & - & -0.08 & -0.001 \\
\hline${ }^{106} \mathrm{Sn}$ & -0.34 & - & -0.13 & -0.002 \\
\hline${ }^{108} \mathrm{Sn}$ & -0.39 & - & -0.14 & -0.002 \\
\hline${ }^{110} \mathrm{Sn}$ & -0.50 & - & -0.17 & -0.003 \\
\hline${ }^{112} \mathrm{Sn}$ & -0.45 & $-0.03(11)$ & -0.15 & -0.003 \\
\hline${ }^{114} \mathrm{Sn}$ & -0.28 & $\begin{array}{c}0.32(3) \\
0.36(4)\end{array}$ & -0.09 & -0.004 \\
\hline${ }^{116} \mathrm{Sn}$ & -0.12 & $\begin{array}{l}-0.17(4) \\
+0.08(8)\end{array}$ & -0.03 & -0.003 \\
\hline${ }^{118} \mathrm{Sn}$ & -0.01 & $-0.05(14)$ & 0.01 & -0.003 \\
\hline${ }^{120} \mathrm{Sn}$ & 0.04 & $\begin{array}{c}+0.022(10) \\
-0.05(10)\end{array}$ & 0.03 & -0.003 \\
\hline${ }^{122} \mathrm{Sn}$ & 0.01 & $\begin{array}{l}-0.28<Q \\
Q<+0.14\end{array}$ & 0.02 & -0.003 \\
\hline${ }^{124} \mathrm{Sn}$ & -0.07 & $0.0(2)$ & -0.01 & -0.003 \\
\hline${ }^{126} \mathrm{Sn}$ & -0.13 & - & -0.04 & -0.002 \\
\hline${ }^{128} \mathrm{Sn}$ & -0.14 & - & -0.05 & -0.002 \\
\hline${ }^{130} \mathrm{Sn}$ & -0.07 & - & -0.03 & -0.001 \\
\hline${ }^{132} \mathrm{Sn}$ & 0.04 & - & 0.05 & 0.015 \\
\hline${ }^{134} \mathrm{Sn}$ & -0.01 & - & 0.00 & -0.001 \\
\hline${ }^{190} \mathrm{~Pb}$ & -0.92 & - & -0.30 & -0.008 \\
\hline${ }^{192} \mathrm{~Pb}$ & -1.15 & - & -0.38 & -0.008 \\
\hline${ }^{194} \mathrm{~Pb}$ & -1.31 & - & -0.44 & -0.008 \\
\hline${ }^{196} \mathrm{~Pb}$ & -1.26 & - & -0.42 & -0.008 \\
\hline${ }^{198} \mathrm{~Pb}$ & -1.05 & - & -0.35 & -0.008 \\
\hline${ }^{200} \mathrm{~Pb}$ & -0.52 & - & -0.17 & -0.006 \\
\hline${ }^{202} \mathrm{~Pb}$ & -0.15 & - & -0.03 & -0.005 \\
\hline${ }^{204} \mathrm{~Pb}$ & 0.10 & $+0.23(9)$ & 0.06 & -0.003 \\
\hline${ }^{206} \mathrm{~Pb}$ & 0.09 & $+0.05(9)$ & 0.06 & -0.002 \\
\hline${ }^{208} \mathrm{~Pb}$ & 0.05 & $-0.7(3)$ & 0.07 & 0.043 \\
\hline
\end{tabular}

$E_{\max }$ and have found that the value of $E_{\max }=100 \mathrm{MeV}$ ensures $1 \%$ accuracy. To calculate the quantities $\mathrm{V}$ and $g_{L}$, the results of Ref. [4] have been used where all the calculations were performed in the coordinate representation using the same self-consistent DF3-a basis as in the present calculation of the matrix element $M_{L L}$. Thus, the single-particle continuum is taken into account adequately in the present calculations. The contribution to the $\mathrm{Q}$ values of the term with $\delta \mathcal{F}$ in figure 3, Eq. (17), turned out to be rather small, $(-(0.01 \div 0.03)$ e b). However, there are cases where these corrections are comparable with the total $Q\left(2_{1}^{+}\right)$value when the proton and neutron values almost compensate each other, e.g. in ${ }^{118} \mathrm{Sn}$ and ${ }^{122} \mathrm{Sn}$ nuclei. The term with $\delta_{L} \mathcal{F}^{\xi}$ contains the anomalous analogs of the corresponding quantities in Eq. (17).

The results are given in Table 4 and figure 4. Except for ${ }^{112} \mathrm{Sn}$ and ${ }^{208} \mathrm{~Pb}$ nuclei, we obtained a reasonable agreement with experimental data [1]. The contribution of the GSC term turned out to be large. Often it is more than $50 \div 60 \%$ of all triangle contributions (column $\mathrm{Q}(\mathrm{GSC}=0)$ )) The usual QRPA (GSC $=0$ and $V=V^{0}$ ), see the last column in Table 4, results in considerably less $Q$ values. 


\section{Quadrupole moments of odd-odd near magic nuclei}

As the odd-odd nuclei are more complicated objects than the odd ones, we consider here only the near-magic oddodd nuclei. Within the above-described self-consistent EDF approach, we calculated the ground state quadrupole moments of odd-odd near-magic nuclei with the use of the approximation disregarding the interaction between two odd particles. This simple approximation can be checked in a pure phenomenological way $[11,28]$ and it turned out reasonable. Within this approximation, the problem is reduced to calculations of quadrupole moments of corresponding odd nuclei.

Indeed, if we neglect the interaction between two quasi-particles, the quadrupole moment of the odd-odd nucleus with the spin $I$ is as follows:

$$
Q_{I}=<I I\left|V^{p}+V^{n}\right| I I>,
$$

where $\Psi_{I I}=\Sigma \varphi_{1} \varphi_{2}<j_{1} m_{1} j_{2} m_{2} \mid I I>$, for the particleparticle case. Here $\varphi_{1}$ is the single-particle wave function with the quantum numbers $1 \equiv \lambda_{1} \equiv\left(n_{1}, j_{1}, l_{1}, m_{1}\right)$. Then the expression for ground state quadrupole moment of the odd-odd near-magic nucleus has the form:

$$
\begin{array}{r}
Q_{I}=(2 I+1)\left(\begin{array}{ccc}
I & 2 & I \\
I & 0 & -I
\end{array}\right)(-1)^{j_{p}+j_{n}+I+2} \times \\
\times\left[\left\{\begin{array}{ccc}
j_{p} & I & j_{n} \\
I & j_{p} & 2
\end{array}\right\} c_{j_{p}}^{-1} Q^{p}+\left\{\begin{array}{ccc}
j_{n} & I & j_{p} \\
I & j_{n} & 2
\end{array}\right\} c_{j_{n}}^{-1} Q^{n}\right],
\end{array}
$$

where $3 \mathrm{j}$-symbol $c_{j}=2 j(2 j-1)^{1 / 2}[(2 j+3)(2 j+2) \times$ $\times(2 j+1) 2 j]^{-1 / 2}, Q^{p}$ and $Q^{n}$ are the quadrupole moments of corresponding odd nuclei. Similar formulae can be easily obtained for the hole-hole, particle-hole and holeparticle cases. The details can be found in the Poster article by Voitenkov et al. [11] at the given conference.

The results of the calculations are presented in Table 5. In column $\mathrm{Q}_{\text {eff }}$ we show the results for the odd-odd nuclei under consideration obtained with the effective charges $e_{e f f}^{p}=2, e_{e f f}^{n}=1$ in order to compare them with this wellknown phenomenological description. We see that there are only three experimental $\mathrm{Q}$ values for the long-living nuclei and our approach describes them rather satisfactory. Other nuclei are short-living ones and our results give reliable predictions for their Q values.

\section{Conclusion}

Quadrupole moments of odd neighbors of semi-magic lead and tin isotopes and $N=50, N=82$ isotones are calculated within the self-consistent TFFS based on the Energy Density Functional by Fayans et al. with the DF3-a parameters fixed previously. The same approach has been used to calculate quadrupole moments of the first $2^{+}$state in tin and lead isotopes as well as the moments of near-magic odd-odd nuclei.

For the quadrupole moments of odd and odd-odd near magic nuclei a good agreement with the experiment has been obtained.For the case of semi-magic nuclei a reasonable agreement with experiment for the quadrupole moments has been obtained for the most part of nuclei considered. In this case when the odd particle belongs to the
Table 5. Quadrupole moments $Q$ (e b) of odd-odd near-magic nuclei.

\begin{tabular}{lccccc}
\hline \hline nucl. & $J^{\pi}$ & $T_{1 / 2}$ & $Q_{\text {eff }}$ & $Q_{\text {theor }}$ & $Q_{\text {exp }}$ \\
\hline${ }_{27}^{54} \mathrm{Co}_{27}$ & $0^{+}$ & $193.28 \mathrm{~ms}$ & - & - & - \\
${ }_{56} \mathrm{Co}_{29}$ & $4^{+}$ & $77.236 \mathrm{~d}$ & 0.19 & 0.30 & $+0.25(9)$ \\
${ }_{56}^{56} \mathrm{Cu}_{27}$ & $\left(4^{+}\right)$ & $93 \mathrm{~ms}$ & 0.14 & 0.28 & - \\
${ }_{29}^{58} \mathrm{Cu}_{29}$ & $1^{+}$ & $3.204 \mathrm{~s}$ & 0.09 & 0.15 & - \\
${ }_{78}^{78} \mathrm{Cu}_{49}$ & $\left(3^{-}\right)$ & $637 \mathrm{~s}$ & -0.18 & -0.21 & - \\
${ }_{29}$ & $\left(4^{-}\right)$ & & $4 \times 10^{-5}$ & -0.03 & - \\
& & 0.24 & 0.21 & - \\
${ }_{49}^{100} \mathrm{In}_{51}$ & $\left(6^{+}\right)$ & $5.9 \mathrm{~s}$ & 0.24 & - \\
${ }_{19}^{13} \mathrm{In}_{81}$ & $1^{-}$ & $0.29 \mathrm{~s}$ & -0.08 & -0.07 & - \\
${ }_{132}^{432} \mathrm{In}_{83}$ & $\left(7^{-}\right)$ & $0.207 \mathrm{~s}$ & -0.40 & -0.29 & - \\
${ }_{49}^{132} \mathrm{Sb}_{81}$ & $(4)^{+}$ & $2.79 \mathrm{~m}$ & -0.30 & -0.22 & - \\
${ }_{514}^{134} \mathrm{Sb}_{83}$ & $\left(0^{-}\right)$ & $0.78 \mathrm{~s}$ & - & - & - \\
${ }_{51}^{206} \mathrm{Tl}_{125}$ & $0^{-}$ & - & - & - & - \\
${ }_{81}^{208} \mathrm{Tl}_{127}$ & $5^{+}$ & $3.053 \mathrm{~m}$ & -0.30 & -0.27 & - \\
${ }_{81}^{208} \mathrm{Bi}_{125}$ & $5^{+}$ & $3.68 \mathrm{E}+5 \mathrm{y}$ & -0.51 & -0.35 & $-0.64(6)$ \\
${ }_{83}^{210} \mathrm{Bi}_{127}$ & $1^{-}$ & $5.012 \mathrm{~d}$ & 0.21 & 0.16 & $+0.136(1)$ \\
${ }_{83}$ & & & & & \\
\hline \hline
\end{tabular}

superfluid subsystem, the Bogolyubov factor $\left(u_{\lambda}^{2}-v_{\lambda}^{2}\right)=$ $\left(\varepsilon_{\lambda}-\mu\right) / E_{\lambda}$ comes to the quadrupole moment value, in addition to the matrix element of the effective field $V_{\lambda}$. This factor makes the quadrupole moment value very sensitive to calculation accuracy of the single-particle energy $\varepsilon_{\lambda}$ of the state under consideration, especially near the Fermi surface as the quantity $Q_{\lambda}$ vanishes at $\varepsilon_{\lambda}=\mu$. For such a situation, influence of the coupling of single-particle degrees of freedom with phonons, see $[5,6,20]$, should be especially important.

For the quadrupole moments of the first $2^{+}$states, we have obtained a noticeable difference from the traditional QRPA approach. In particular, new terms with $\delta_{L} \mathcal{F}$ and $\delta_{L} \mathcal{F}^{\xi}$ appear, which contain the density derivatives of both the ph and pp effective interactions. In the problem under consideration, their contribution turned out to be rather small, as a rule. However, for consistency, these terms should be included. Except for the ${ }^{112} \mathrm{Sn}$ and ${ }^{208} \mathrm{~Pb}$ cases, a reasonable agreement has been obtained with the experiment available. Using the self-consistent method which contains no newly adjusted parameters we have also predicted the values of quadrupole moments of the first $2^{+}$ states in several unstable lead and tin isotopes including the ${ }^{100} \mathrm{Sn}$ and ${ }^{132} \mathrm{Sn}$ nuclei. An unexpectedly large contribution of ground state correlations to the $Q\left(2_{1}^{+}\right)$values is found. A non-trivial dependence of the quadrupole moments of the first $2^{+}$states on the neutron excess is found which can be traced to the negative proton contributions. A similar behavior could probably be present in other isotope chains.

Quadrupole moments of unstable nuclei including those near the exotic ${ }^{100} \mathrm{Sn}$ and ${ }^{132} \mathrm{Sn}{ }^{56,78} \mathrm{Ni}$ are also predicted, which should be of special interest.

\section{Acknowledgements}

The work was partly supported by the DFG and RFBR Grants Nos.436RUS113/994/0-1 and 09-02-91352NNIOa, by the Grants NSh-7235.2010.2 and 2.1.1/4540 of the Russian Ministry for Science and Education, and by the RFBR grants 11-02-00467-a and 12-02-00955-a. 


\section{References}

1. N. Stone, At. Data \& Nucl. Data Tables 90, 75 (2005)

2. V. Khodel, E.E. Saperstein, Phys. Rep. 92, 183 (1982)

3. S.A. Fayans, S.V. Tolokonnikov, E.L. Trykov, and D.

Zawischa, Nucl. Phys. A 676, 49 (2000)

4. S.V. Tolokonnikov, S. Kamerdzhiev, D. Voitenkov, S. Krewald, and E.E. Saperstein, Phys. Rev. C 84, 064324 (2011)

5. S.V. Tolokonnikov, S. Kamerdzhiev, D. Voitenkov, S. Krewald, and E.E. Saperstein, Eur. Phys. J A 48:70 (2012)

6. I.N. Borzov, S.V. Tolokonnikov, and E.E. Saperstein, Phys. At. Nucl. 71, 469 (2008)

7. I.N. Borzov, E.E. Saperstein, S.V. Tolokonnikov, G. Neyens, and N. Severijns, Eur. Phys. J. A 45, 159 (2010)

8. S.V. Tolokonnikov, E.E. Saperstein, Phys. At. Nucl. 73, 1684 (2010)

9. A.B. Migdal, Theory of finite Fermi systems and applications to atomic nuclei (Wiley, New York, 1967)

10. D. Voitenkov, S. Kamerdzhiev, S. Krewald, E.E. Saperstein, S.V. Tolokonnikov, Phys. Rev C 85, 054319 (2012)

11. D. Voitenkov, O. Achakovskiy, S. Kamerdzhiev, and S.V. Tolokonnikov, Poster at the NSRT12 Conference.

12. W. Kohn and L. J. Sham, Phys. Rev. A 140, 1133 (1965)

13. M. Baldo, U. Lombardo, E.E. Saperstein, M.V. Zverev, Phys. Rep. 391 261, (2004)

14. V.G. Soloviev, Theory of Complex Niclei, (Oxford: Pergamon Press, 1976)

15. A. Bohr and B.R. Mottelson, Nuclear Structure (Benjamin, New York, Amsterdam, 1969.), Vol. 1

16. S.P. Kamerdzhiev, Sov. J. Nucl. Phys. 2, 415 (1965)

17. S.P. Kamerdzhiev, Sov. J. Nucl. Phys. 9, 324 (1969)

18. V.A. Khodel, Sov. J. Nucl. Phys. 24, 376 (1976)

19. S. Kamerdzhiev, E.E. Saperstein, Eur. Phys. J. A 37, 159 (2008)

20. S.P. Kamerdzhiev, A.V. Avdeenkov, D.A. Voitenkov, Phys. At. Nucl. 74, 1478 (2011)

21. J. Speth, Z. Phys. 239, 249 (1970)

22. B.L. Birbrair, Phys. Lett. B 32, 165 (1970)

23. S. Kamerdzhiev, D. Voitenkov, in Proceedings of the XII Intl. Seminar on Electromagnetic Interactions of Nuclei (EMIN-2009) (Moscow, Russia, September 17-19, 2009), INR RAS, Moscow, 2010, p. 68; arXiv:1110.0654[nucl-th] (2011)

24. V.Yu. Ponomarev, Ch. Stoyanov, N. Tsoneva, M. Grinberg, Nucl. Phys. A 635, 470 (1998)

25. R.A. Broglia, R. Liotta, and V. Paar, Phys. Lett. B 38, 480 (1972)

26. A.I. Vdovin, Ch. Stoyanov, Izv. Akad. Nauk SSSR, Ser. Fiz., 38, 2598 (1974)

27. A.I. Vdovin, Ch. Stoyanov, Izv. Akad. Nauk SSSR, Ser. Fiz., 38, 2604 (1974)

28. S.P. Kamerdzhiev, O.I. Achakovskiy, D.A. Voitenkov, and S.V. Tolokonnikov, Phys. At. Nucl., to be published 